\title{
Anabases
}

ANABASES Traditions et réceptions de l'Antiquité

$16 \mid 2012$

Varia

\section{Humanistes et antiquaires. Le De Asse de Guillaume Budé}

\section{Luigi-Alberto Sanchi}

\section{(2) OpenEdition}

1 Journals

Édition électronique

URL : http://journals.openedition.org/anabases/3977

DOI : 10.4000/anabases.3977

ISSN : 2256-9421

Éditeur

E.R.A.S.M.E.

\section{Édition imprimée}

Date de publication : 1 octobre 2012

Pagination : 207-223

ISSN : 1774-4296

\section{Référence électronique}

Luigi-Alberto Sanchi, « Humanistes et antiquaires. Le De Asse de Guillaume Budé », Anabases [En ligne] 16 | 2012, mis en ligne le 01 octobre 2015, consulté le 20 octobre 2019. URL : http:// journals.openedition.org/anabases/3977; DOI : 10.4000/anabases.3977 
Anabases 16 (2012), p. 207-223.

\section{Humanistes et antiquaires Le De Asse de Guillaume Budé}

Luigi-Alberto SANCHI

À la Grèce.

Parus en mars 1515, les Cinq livres de l'As et ses fractions ou De Asse et partibus eius libri quinque de Guillaume Budé (1468-1540) ${ }^{1}$ sont précédés d'une préface dont l'intitulé, Ad optimum quemque et candidissimum uirum litterarum et philosophie studio-

1 Je remercie pour leurs encouragements, conseils et réflexions - aides précieuses à la rédaction du présent article - Jean-François Maillard, Pascal Payen, Perrine Galand, Olivier Pédeflous et Luigi Ferreri. Sur Budé, voir les monographies suivantes, dans l'ordre chronologique : Daniel ReBitTÉ, Guillaume Budé, restaurateur des études grecques en France. Essai historique [1846], réimpr. Genève, Slatkine, 1969 ; Eugène DE BuDÉ, Vie de Guillaume Budé (1467-1540), fondateur du Collège de France, Paris, D. É. Perrin, 1884 (disponible sur internet par le système "print on demand") ; Louis DelaruelLe, Guillaume Budé. Les origines, les débuts, les idées maîtresses [1907], réimpr. Genève, Slatkine, 2012 ; Jean Plattard, Guillaume Budé (1468-1540) et les origines de l'humanisme français [1923], réimpr. Paris, Les Belles Lettres, 1966 ; David O. McNeill, Guillaume Budé and Humanism in the Reign of Francis I, Genève, Droz, 1975 ; Marie-Madeleine DE LA GARANDERIE, Christianisme et lettres profanes. Essai sur l'humanisme français (1515-1535) et sur la pensée de Guillaume Budé, Paris, H. Champion, 1995 [1976] ; Gilbert GAdOFfre, La Révolution culturelle dans la France des humanistes. Guillaume Budé et François I ${ }^{e r}$, Genève, Droz, 1997 ; Guy Gueudet, L'art de la lettre humaniste, Paris, H. Champion, 2004 ; Luigi-Alberto SANCHI, Les "Commentaires de la langue grecque" de G. Budé. L'ouvre, ses sources, sa préparation, Genève, Droz, 2006 ; Sylvie ChARTON Le CleCh, Guillaume Budé : l'humaniste et le prince, Paris, Riveneuve, 2008 ; Louise KaTz, Guillaume Budé et l'art de la lecture, Turnhout - Bruxelles, Brepols - Musée de la Maison d'Érasme, 2009 ; M.-M. DE La Garanderie, Guillaume Budé, philosophe de la culture [recueil d'articles 1964-2005], Paris, Classiques Garnier, 2010. Ce dernier ouvrage comporte une mise à jour bibliogra- 
sum, affiche le souhait de s'adresser - au lieu d'un dédicataire plus ou moins puissant - à un public humaniste aussi large que possible. Cette préface annonce en outre un programme bien plus vaste que «l'as " promis dans le titre. En effet, après la mention de son ouvrage précédent, les Annotationes in XXIV Pandectarum libros qui ont renouvelé en 1508 l'exégèse des sources du droit romain, l'auteur affirme ceci :

Nunc alteram lucubrationem grandioris incepti operosiorisque profero, non in unum genus illam scriptorum editam, sed in uniuersum pertinentem ad Antiquitatis interpretationem et per omne prope genus auctorum probiorum utraque lingua patentem.

Je présente maintenant une deuxième étude de plus grande ampleur que le premier projet : elle ne porte plus sur une seule discipline, mais concerne l'interprétation de l'Antiquité en général et s'ouvre sur presque tous les genres pratiqués par les bons auteurs dans les deux langues.

Point d'exagération rhétorique : comme on le verra plus loin, Budé a tenu sa promesse.

Pour aborder convenablement cet ouvrage très dense, il faudrait au préalable pallier une double absence dans la panoplie théorique actuelle. D'une part, l'apport intellectuel des Antiquaires est, dans son ensemble, relativement méconnu dans le débat historiographique français en dehors du cercle étroit des spécialistes. D'autre part, l'œuvre de Guillaume Budé en particulier, ainsi que son héritage scientifique touchant notamment la recherche érudite sur l'Antiquité - n'est guère présent au sein des études sur la tradition humaniste et antiquaire, courant qui s'est d'ailleurs structuré bien après notre auteur, surtout aux XVII ${ }^{\mathrm{e}}$ et XVIII ${ }^{\mathrm{e}}$ siècles. Une telle absence s'explique sans doute pour partie par le manque d'une édition critique moderne du De Asse, surtout connu encore aujourd'hui à travers l'étude méthodique qu'en a donnée en 1907 Louis Delaruelle ${ }^{2}$. Imparfaitement saisie par ce dernier, la profonde originalité de

phique des travaux sur Budé, comprenant de nombreux articles que nous n'aurons pas l'occasion de citer ici.

2 L. Delaruelle, Guillaume Budé, p. 130-198. Outre de fréquentes mentions dans la bibliographie déjà citée, le De Asse a été ensuite l'objet des réflexions - se rattachant à notre propos au moins en partie - de G. Gadoffre, "Guillaume Budé e la storia di Roma ", Studi romani, 35 (1987), p. 263-276 ; de M.-M. DE LA Garanderie, surtout dans "L'harmonie secrète du De Asse de Guillaume Budé " [1968], in Guillaume Budé, p. 269-284, dans "Guillaume Budé, lecteur de Pline l'Ancien " [1997-2000], ibid., p. 285-301, dans Christianisme et lettres profanes, "La philosophie du De Asse ", p. 285-305 ; de Guy Lavoie, "Y a-t-il un secret dans l'architecture du De Asse?", Renaissance \& Réforme, 3, 1 (1979), p. 30-44 ; de Jean CÉARD, "La transformation du genre du commentaire ", dans L'Automne de la Renaissance, 1580-1630 (actes coll. Tours, 1979), Paris, Vrin, 1981, p. 101-115 ; de Jean-Claude Margolin, " De la digression au commentaire : pour une lecture humaniste du De Asse de Guillaume Budé ", in Neo 
l'œuvre de Budé mérite néanmoins toute l'attention des historiens afin de lui redonner toute sa place à l'intérieur des études sur l'antiquaire comme dans le débat sur l'historiographie. C'est l'enjeu de la présente contribution.

Considérons d'abord le status quastionis à ce propos. On pourrait prendre à témoin un riche ouvrage collectif récemment paru, Historiographies. Concepts et débats ${ }^{3}$, qui se propose de rendre compte de l'écriture et des méthodes en usage aujourd'hui en histoire. À défaut d'une section consacrée à l'Humanisme proprement dit ${ }^{4}$, il ne réserve que quinze pages, sur quelque mille trois cents, à l'entrée Renaissance, notion étudiée surtout - et à juste titre - pour la problématiser. Dans cet article, par exemple, les

Latin and Vernacular in Renaissance France, Oxford, Clarendon, 1984, p. 1-25; d'Alain Michel, "L'or dans le De Asse de G. Budé» dans L'or au temps de la Renaissance. Du mythe à l'économie, Paris, Univ. de Paris-Sorbonne, 1978, p. 5-14. Le grand dialogue politicoculturel d'allure cicéronienne entre Budé et son vieil ami François Deloynes que clôt le De Asse, appelé "Épilogue » dès la première édition, a fait l'objet d'études spécifiques qui ont permis, entre autres, de constater l'existence d'importants ajouts de l'auteur au fil des éditions : voir M.-M. De La Garanderie, "Sur quelques pages de l'Épilogue du De Asse ajoutées en 1516 " [1999], Guillaume Budé, p. 317-336, et John. M. HEadLEY, "The problem of Counsel revisited once more : Budé's De Asse (1515) and [Thomas More's] Utopia I (1516) in defining a political moment ", in C. S. CelenzA, K. Gowens (dir.), Humanism and creativity in the Renaissance. Essays in honor of Ronad G. Witt, Leyde, Brill, 2006, p. 141-168. Pour utile qu'elle soit d'un point de vue avant tout littéraire, touchant l'écriture, le style et l'emploi de certains topoi ou éléments philosophiques, cette bibliographie n'est fondée ni sur un dépouillement complet des contenus et des sources du De Asse, ni sur un établissement du texte rigoureux, distinguant les différentes couches rédactionnelles.

3 C. Delacroix, F. Dosse, P. Garcia, N. Offenstadt (dir.), Paris, Gallimard, 2010, deux tomes en pagination continue. Ce vaste effort collectif est un outil de recherche très bienvenu autant qu'un véritable manuel d'initiation à l'historiographie.

4 L'opportunité du terme historiographique (on ne prendra pas en considération ici ses autres sens) d'Humanisme et de celui, proche, de Renaissance, a fait l'objet de débats parfois polémiques et de précisions diverses dont le développement requerrait un article à part et une bibliographie spécifique, que l'on glane en partie dans celle du présent essai. Ce n'est pas esquiver la question que de rappeler les définitions convergentes - et satisfaisantes pour notre but - que donne un ouvrage de référence comme le Dictionnaire de la langue française de Paul Robert (éd. Josette Rey-Debove, Alain Rey, Paris, Le Robert, 2002). À l'entrée humaniste, on lit : "Lettré de la Renaissance qui se consacrait à l'étude des écrivains antiques et en faisait connaître les œuvres et les idées "; l'Humanisme est défini comme "mouvement intellectuel européen de la Renaissance, caractérisé par un effort pour relever la dignité de l'esprit humain et la mettre en valeur, et un retour aux sources gréco-latines »; la Renaissance est à la fois l'" essor intellectuel provoqué, à partir $\mathrm{du} \mathrm{XV}$ siècle en Italie, puis dans toute l'Europe, par le retour aux idées, à l'art antiques gréco-latins " et la "période historique allant du XIV siècle ou du XV $v^{\mathrm{e}}$ siècle à la fin du $\mathrm{XVI}^{\mathrm{e}}$ siècle ». 
alinéas placés sous le titre prometteur d' "Un nouveau rapport au passé » ne comportent aucun développement sur les historiens humanistes 5 .

On glane ailleurs quelques mentions de cet acte historiographique majeur qu'est la réfutation de la Donation de Constantin composée par Laurent Valla en 1444, tour à tour citée dans le cadre des luttes religieuses du XVI ${ }^{\mathrm{e}}$ siècle, car publiée en 1517 par Ulrich von Hutten, ou présentée comme avatar du travail sur les archives ou, encore, mentionnée en tant qu'anachronisme dévoilé par excellence ${ }^{6}$. Dans l'entrée consacrée à l'Histoire économique, en outre, l'hypothèse que Guillaume Budé puisse être le fondateur de cette science est d'emblée écartée sans que soit même citée la contribution concernée, le De Asse justement. C'est d'ailleurs la seule page d'Historiographies où le grand humaniste parisien se trouve nommé 7 .

De manière générale dans cet ouvrage, les auteurs d'avant le XVIII ${ }^{\mathrm{e}}$ siècle ne font malheureusement l'objet que de mentions aussi brèves qu'aléatoires. Choix réducteur, d'autant plus regrettable qu'il semble faire l'impasse sur un domaine historiographique bien vivant, y compris en langue française : outre l'apport novateur d'Anabases dans les dernières années ${ }^{8}$, depuis 1988 l'ouvrage classique de Blandine Barret-Kriegel,

5 Voir Historiographies. Concepts et débats, p. 1172-1175. L'auteur de l'article évoque la vision historique des Vasari et Pétrarque, " procédant d'une réflexion d'origine italienne qui dénonçait le medium avum (Moyen Âge) comme un âge de ténèbres [...] », pour mieux dénoncer à son tour, dès les premières lignes de l'article, les " poncifs " répandus sur la Renaissance par Jakob Burckhardt ou Jules Michelet.

6 Voir ibid., respectivement p. 1230 (Rôle social de l'historien), p. 69-70 (Archives, documents, sources) et p. 665 (Anachronisme; le même auteur revient sur l'épisode dans l'entrée Événement, p. 753). Le nom de Valla apparaît, sans plus, p. $934-935$ (Vérité). Voir L. Valla, La Donation de Constantin, éd. J.-B. Girard, Paris, Les Belles Lettres, 1993.

7 Histoire économique, dans Historiographies, p. 293 : "Certains auteurs font remonter l'histoire économique aux travaux d'Ibn Khaldun [...], à ceux de Guillaume Budé et de Jean Bodin [...]. Mais celle-ci se constitue comme discipline universitaire académique, autonome, reconnue et institutionnalisée seulement vers 1850 [...]. " Perspective bien étroite, qui empêche de voir, a fortiori de prendre en considération, les tentatives précédentes. Le premier grand exemple d'une histoire scientifique de l'économie antique, celle d'August Bєскн, Die Staatshaushaltung der Athener, date de 1817 (réimpr. New York, Elibron Classics, 2 vol., 2005). À propos de Bœckh et de sa querelle méthodologique avec Gottfried Hermann, tenant de la philologie formelle, voir la réflexion fort stimulante qu'en propose Luciano CANFora, "Enzo Degani e la storia degli studi classici ", dans Da Aiẃv a Eikasmos. Atti della giornata di studio sulla figura e l'opera di Enzo Degani, Bologne, Pàtron, 2002, p. 101-108.

8 Le sous-titre Traditions et réceptions de l'Antiquité l'indique d'entrée de jeu. Voir notamment les exposés du projet de recherche de la revue fournis par Pascal PAYEN : «L'Antiquité après l'Antiquité : parcours et détours d'un projet éditorial » et "Réception des historiens anciens et fabrique de l'histoire ", Anabases, 1 (2005), respectivement p. 5-13 et 277-283, ainsi que "L'Antiquité et ses réceptions : un nouvel objet d'histoire ", éditorial du n ${ }^{\circ} 10$ (2009), p. 9-23. 
Les Historiens et la monarchie9 a permis d'évaluer précisément le rôle de l'érudition française au Grand Siècle dans la construction historique de la France ; Alain Schnapp, avec sa vaste étude $L a$ Conquête du passé : aux origines de l'archéologie ${ }^{10}$, a ensuite posé un cadre théorique plus général pour penser le passé des recherches archéologiques à commencer par l'Antiquité elle-même ${ }^{11}$; l'étude de la réception de l'Antiquité a pris des formes variées et toujours pertinentes ${ }^{12}$; des ouvrages généraux et collectifs tels que les deux volumes Lieux de savoir (2007 et 2011) ${ }^{13}$ réservent une place de choix aux institutions et cénacles érudits, voire humanistes.

En amont, la réflexion autour de ce domaine de recherche a été renouvelée de manière décisive en 1950 par Arnaldo Momigliano dans son article fondateur "Ancient History and the Antiquarian ", qui a inspiré les chercheurs jusqu’à nos jours ${ }^{14}$. Les éléments de l'ample analyse de Momigliano sont bien connus ; ils présentent un intérêt de premier ordre pour saisir la formation de la conception moderne du métier d'historien ${ }^{15}$.

9 B. Barret-Kriegel, Les Historiens et la monarchie, 4 vol., Paris, puf, 1988.

10 Paris, Éditions Carré, 1993.

11 Nous renvoyons aux articles cités de P. PAYEN pour un approfondissement de la question et de nombreuses pistes bibliographiques.

12 À titre d'exemple, voir le remarquable travail de Claude Nicolet, La Fabrique d'une nation. La France entre Rome et les Germains, Paris, Perrin, 2003, ou l'essai foisonnant de Pierre Vidal-Naquet, L'Atlantide. Petite histoire d'un mythe platonicien, Paris, Les Belles Lettres, 2005.

13 Lieux de savoir, I : Espaces et communautés, II : Les mains de l'intellect, dir. C. Jacob, Paris, A. Michel, 2007-2011.

14 Journal of the Warburg and Courtauld Institutes, 13 (1950), p. 285-315, recueilli ensuite dans A. Momigliano, Contributo alla storia degli studi classici, Rome, Edizioni di Storia e letteratura, 1955, p. 67-106 ; voir sa traduction française, «L'histoire ancienne et l'Antiquaire ", dans id., Problèmes d'historiographie ancienne et moderne, Paris, Gallimard, 1983, p. 243-299. Riccardo FUBINI en a récemment précisé le contexte et montré qu'il s'agissait en réalité d'une réponse implicite adressée au philosophe italien Benedetto Croce à propos de sa conception de l'histoire politique : voir "All'origine della scienza antiquaria : una paternità da rivedere ", Medioevo e Rinascimento, 22 (n.s. 19) (2008), p. 233-244. Pour une vue d'ensemble de la réception des idées de Momigliano, voir Ingo HerkLotz, "Arnaldo Momigliano's "Ancient History and the Antiquarian" : a critical review ", in Momigliano and Antiquarianism. Foundation of the Modern cultural sciences, Toronto, Toronto U.P., 2007, p. 127-153. Voir aussi l'ensemble de cette dernière publication ainsi que Ancient History and the Antiquarian. Essays in Memory of Arnaldo Momigliano (actes coll. Londres, Warburg Inst., 1991), Londres, Warburg Institute, 1995.

15 La fécondité des réflexions de Momigliano sur ce sujet est prouvée par les fréquentes mentions émaillant la bibliographie, notamment les travaux sur l'Humanisme (parmi lesquels figure une étude sur Budé lecteur d'Homère : "How G. Budé read his Homer ", in Commerce with the Classics. Ancient Books and Renaissance Readers, Ann Arbor, Univ. of Michigan Press, 1997, p. 135-183) menés par Anthony Grafton, l'auteur qui a le plus approfondi cette thématique, mais aussi dans la synthèse de Roberto WeIss, The 
Momigliano commence par une introduction qui marque le tournant des Lumières comme un passage à une nouvelle forme d'humanisme : "In the Eighteenth century a new humanism competed with the traditional one. "Les gentlemen qui s'y adonnent sont ainsi différenciés de leurs devanciers : " [they] preferred travel to emendation of texts and altogether subordinated literary texts to coins, statues, vases and inscriptions ${ }^{16}$." Passage de l'emendatio érudite à l'étude des sources mâtinée d'archéologie qui configure la méthode historique moderne : "The Age of the Antiquaries [...] meant a revolution in historical method. [...] The Age of Antiquaries set standards and posed problems of historical method that we can hardly call obsolete to-day ${ }^{17}$." Momigliano rappelle ici que la distinction entre sources primaires et secondaires ("original and derivative authorities") ne se répand parmi les historiens qu'à la fin du XVII ${ }^{\mathrm{e}}$ siècle. Ce cadre chronologique privilégiant le XVIII ${ }^{\mathrm{e}}$ siècle posé, la partie du raisonnement de Momigliano qui intéresse notre propos est la première, "The origins of Antiquarian research ". Comme on le sait, il fait remonter ce genre d'études à l'Antiquité même ${ }^{18}$, depuis la Grèce classique jusqu'au grand modèle varronien, mais accorde ensuite une attention particulière à l'Humanisme, à Pétrarque et surtout à l'œuvre capitale de Flavio Biondo (1392-1463) : "Roma triumphans has already the fourfold classification which many later handbooks preserve under the heading : antiquitates publicæ, privatæ, sacræ, militares. " Les contributions philologiques et en même temps antiquaires du Politien des Miscellanées (1489) et de Colius Rhodiginus (Antiqua lectiones, 1516) sont mentionnées en note de bas ${ }^{19}$, où le nom de Budé ne figure pas, non plus que dans les pages suivantes où

Renaissance discovery of classical Antiquity, Oxford, Blackwell, 1969, importante pour notre propos ; voir aussi la traduction italienne, La scoperta dell'Antichità classica nel Rinascimento, Padoue, Antenore, 1989. Blandine BARRET-Kriegel reconnaît sa dette envers Momigliano dans La Défaite de l'érudition, tome II des Historiens et la Monarchie, op. cit., p. 17-22, au chapitre Premier, "La source, l'archive, le diplôme ", allant jusqu'à donner la traduction d'un passage-clé de "L'histoire ancienne et l'Antiquaire ". Sur les brisées de ce grand historien, voir également l'essai très fouillé et suggestif de Carlo GinZBuRG, "Description et citation » ("Ekphrasis and quotation », 1988, en traduction française dans le recueil - dédié à la mémoire de Momigliano - Le Fil et les traces. Vrai faux fictif [2006], Paris, Verdier, 2010, p. 23-60), où l'auteur esquisse une histoire du scepticisme historiographique avant La Mothe La Vayer, dès Francesco Robortello (1548) voire encore plus tôt, dans une lettre de Manuel Chrysoloras datant de 1411 ; à compléter par l'Introduction du même GinzBURG à l'édition française citée de L. VALLA, La Donation de Constantin, où il pose la problématique de la preuve en histoire en partant de la théorie rhétorique de l'Antiquité à la Renaissance. Voir enfin les pages de Vincenzo FERA, "Problemi e percorsi della ricezione umanistica ", dans Lo Spazio letterario di Roma antica, t. III. La Ricezione del testo, Rome, Salerno Ed., 1990, p. 513-543, qui se situent d'emblée dans le sillon de Momigliano.

16 A. Momigliano, Contributo, p. 67.

17 Op. cit., p. 68.

18 Op. cit., p. 69-73.

19 Op. cit., p. 73, note 14. 
Momigliano évoque les travaux érudits de la génération successive, avec Carlo Sigonio, Fulvio Orsini, Juste Lipse, notant que le terme varronien d'Antiquitates revient surtout avec Rosinus (Johannes Rossfeld), auteur en 1583 de l'Antiquitatum Romanarum corpus absolutissimum. Concernant l'activité de ces savants, Momigliano note qu'ils ne se concevaient pas comme des historiens : "They commented historians and supplemented historians, but usually did not claim to be historians ${ }^{20}$. "La raison invoquée met en cause le genre adopté : "They modelled themselves on Biondo's Roma triumphans which was not a history, but a systematic survey ${ }^{21}$. "Nous n'allons pas parcourir les étapes ultérieures de cette grande fresque historiographique ; qu'il suffise ici de souligner à quel point cette manière de présenter l'évolution des recherches savantes se focalisant sur les grands siècles de l'Antiquaire, parfaitement justifiée au demeurant, a d'une part orienté les travaux à venir et d'autre part rejeté involontairement dans une zone de pénombre plusieurs auteurs intéressants, issus du Quattrocento comme François Filelfe (1398-1481) ou Giulio Pomponio Leto $(1428-1497)^{22}$, ou appartenant à l'époque immédiatement suivante, celle de Budé et du premier humanisme français ${ }^{23}$.

Le nom de Budé est cependant trop important pour en faire l'impasse fût-ce en parlant d'historiographie. Parmi les ouvrages généralistes en langue française, c'est à ma connaissance le manuel de Jean-Maurice Bizière et Pierre Vayssière ${ }^{24}$ qui a le mieux rendu compte de la nouveauté du De Asse, dans une présentation brève mais éloquente :

C'est de l'érudition et, plus particulièrement, de Guillaume Budé (1468-1540) que l'histoire nouvelle devait tirer ses éléments constitutifs initiaux. [...] il montra dans son De Asse, publié en 1515, qu'une analyse philologique de textes non plus littéraires mais renvoyant aux aspects les plus concrets des relations sociales, comme ceux liés à la monnaie ou au droit, permettrait de renouveler fondamentalement la connaissance du passé en même temps qu'elle ouvrait des champs jusque-là inexplorés à ceux qui voulaient consacrer leurs efforts à ce type de recherches.

20 Op. cit., p. 75.

21 Ibid.

22 C'est ce qu'ont - entre autres - précisé les recherches menées dans le sillage de Momigliano : voir R. FubinI, "All'origine della scienza antiquaria ", p. 239-240 (sur Leto et sur son élève Andrea Fulvio) et, dans le recueil cité Ancient history... Essays in memory of $A$. M., Jean-Louis FERRARY, "Naissance d'un aspect de la recherche antiquaire. Les premiers travaux sur les lois romaines: de l'Epistula ad Cornelium [1439] de Filelfo à l'Historia iuris ciuilis [1515] d'Aymar du Rivail ", p. 33-72.

23 Voir toutefois, dans le recueil cité Ancient history... Essays in memory of A. M., p. 73-103, l'article de Carlo DionisotTi sur «Claude de Seyssel » [v. 1450-1520] où il est par ailleurs question de Budé, incidentalement.

24 J.-M. Bizİ̀re et P. VAyssière, Histoire et historiens. Antiquité, Moyen Âge, France moderne et contemporaine, Paris, Hachette, 1995, p. 83. Le chapitre concerné a pour titre «La révolution historique du XVI ${ }^{\mathrm{e}}$ siècle ». 
Ces quelques lignes indiquent de manière pertinente le rôle qu'il convient d'assigner au De Asse dans le cheminement de l'historiographie moderne. C'est également la conclusion à laquelle parvient un ouvrage de synthèse aussi utile à notre propos qu'assez peu cité dans le domaine francophone, Foundations of modern historical scholarship : language, law and history in the French Renaissance, écrit par Donal R. Kelley ${ }^{25}$, qui place Guillaume Budé au centre du parcours menant de l'Humanisme italien à la grande époque des historiens robins dans la France des guerres de Religion ${ }^{26}$. Ayant toutefois pour objet l'humanisme juridique, Kelley bâtit sur les Annotationes in Pandectas plutôt que sur le De Asse l'essentiel de son analyse de la contribution de notre humaniste aux progrès de la recherche en histoire ${ }^{27}$. La fortune moderne du De Asse de Budé est certes un peu mieux attestée lorsqu'on la cherche du côté de la numismatique $^{28}$. Roberto Weiss, dans un chapitre de son The Renaissance discovery of classical Antiquity consacré à celle-ci, reconnaît pleinement les mérites du De Asse tant sur le plan philologique que sur celui de la numismatique naissante et en réitère la portée dans la conclusion du livre ${ }^{29}$.

25 New York, Columbia U. P., 1970.

26 Voir aussi, à ce propos, L.-A. SANCHI, «L'eredità intellettuale di G. Budé negli storici francesi del tardo Cinquecento ", Quaderni di storia, 59 (2004), p. 59-77 (= M. FIrPo [dir.], Nunc alia tempora, alii mores. Storici e storia in età post-tridentina, Florence, Olschki, 2005, p. 529-540).

27 D.R. Kelley, p. 63-80. Pour une tentative récente d'aborder la richesse des Annotations aux Pandectes de Budé, voir Gerald SANDY, "Guillaume Budé : philologist and polymath. A preliminary study ", in G. SANDY (dir.), The classical heritage in France, Leyde, E.J. Brill, 2002, p. 79-108.

28 Pour quelques titres où le De Asse est présenté sur la base de renseignements de deuxième main, mais utilement inséré dans un contexte cohérent d'études érudites : voir Elvira Eliza Clain-Stefanelli, Numismatics, an ancient science. A survey of its history, Washington, (s. n.), 1965, en particulier p. 18-19 ; Richard COOPER, "Collectors of coins and numismatic scholarship in early Renaissance France ", in M. H. Crawford, C. R. Ligota, J. R. Trapp (dir.), Medals and coins. From Budé to Mommsen, Londres, The Warburg Inst., 1990, p. 5-23 ; Jean-Baptiste GIARD, "Critique de la science des monnaies antiques », Journal des savants, 3 (1980), p. 225-245. Voir en outre une référence très récente au De Asse dans la préface d'Andrew Burnett à l'étude de Federica Missere Fontana, Testimoni parlanti. Le monete antiche a Roma tra Cinquecento e Seicento, Rome, Quasar, 2009, p. 13, mais aussi à l'intérieur de ce volume, p. 364 à propos des sources d'Enea Vico, auteur en 1555 et en 1558 des Discorsi sopra le medaglie degli antichi. Cette ouvre a peut-être préparé le terrain à la parution successive, en 1562 à Florence, du Trattato delle monete e valuta loro, ridotte dal costume antico all'uso moderno, di M. Guglielmo Budeo, traduction italienne abrégée du De Asse, par Giovan Bernardo Gualandi.

29 Voir The Renaissance discovery, respectivement p. 167-179 et 203-206 (trad. it. citée, p. $195-210$ et 238-243). 
Si l'on adopte la distinction qu'établit Momigliano à l'orée de l'essai déjà cité entre l'Humanisme proprement dit et ce " new Humanism " qu'est l'antiquaire, comment caractériser alors l'apport du premier ? La réponse à cette question nous amène à compléter le tableau tracé jusqu'ici en y plaçant l'élément central, l'Humanisme, à l'aune duquel il convient d'évaluer le De Asse et son évolution de 1515 à l'édition définitive de 1541.

Jugée préscientifique au sein des études classiques, la période humaniste est de surcroît marquée par la convergence de méthodes et démarches de recherche désormais séparées et indépendantes les unes des autres. Vices qui compliquent la tâche de présenter les travaux des humanistes aux historiens d'aujourd'hui : les résultats de cette historiographie seraient d'autant moins utilisables qu'il semble - à tort ou à raison malaisé de les traduire dans les catégories pratiquées de nos jours. Or la richesse et la variété d'expériences historiographiques en matière de réception de l'Antiquité que l'on constate à la Renaissance, depuis son éclosion italienne au cours du Quattrocento ${ }^{30}$ notamment au contact des immigrés grecs ${ }^{31}$ - jusqu'à son expansion européenne au $\mathrm{XVI}^{\mathrm{e}}$ siècle, n'ont pas d'égales à d'autres époques. Considérons en un regard rapide quelques grands axes d'investigation humaniste touchant l'Antiquité ${ }^{32}$ et ses sources ${ }^{33}$ :

30 On connaît les précurseurs, surtout François Pétrarque qui, en plein XIV siècle, tâchait par exemple de réunir en un seul manuscrit, l'actuel Harleianus 2493 de la British Library, les Décades de Tite-Live recueillies au cours de ses recherches. Pour une excellente présentation d'ensemble de l'" Humanisme italien de Pétrarque à Valla " voir, en attendant une traduction française, G. Cappelli, L'Umanesimo italiano da Petrarca a Valla, Rome, Carocci, 2010 (nouvelle édition augmentée ; première édition, en espagnol : El Humanismo italiano. Un capitulo de la cultura europea entre Petrarca y Valla, Madrid, Alianza Editorial, 2007).

31 Voir à ce sujet les ouvrages de Nigel G. WiLson, Da Bisanzio all'Italia. Gli studi greci nell'Umanesimo italiano, Alessandria, Ed. dell'Orso, 2000 (édition italienne de référence, revue et augmentée après le compte-rendu d'Anna PonTANI, Thesaurismata, 25 (1995), p. 83-125, de From Byzantium to Italy. Greek Studies in the Italian Renaissance [1992]), et de J. Harris, Greek Emigres in the West, 1400-1520, Camberley, Porphyrogenitus, 1995.

32 Capitale dans leur découverte du passé, l'Antiquité est loin de constituer l'unique préoccupation historique des humanistes. Voir à ce propos A. Schnapp, La Conquête du passé, p. 104 et sq., et D. R. Kelley, The Foundations of modern historical scholarship, auxquels l'on ajoutera, pour la position du problème du point de vue de l'histoire de la philosophie, Marie-Dominique Couzinet, Histoire et méthode à la Renaissance. Une lecture de la Methodus ad facilem historiarum cognitionem de Jean Bodin, Paris, Vrin, 1997.

33 Nous signalons dans les notes à suivre quelques ouvrages favorisant une première connaissance des sujets évoqués. 
- une quête systématique des manuscrits-sources grecs et latins - historiques ou autres - dans le but d'en procurer des éditions complètes, voire de découvrir des auteurs ou des œuvres encore inconnus ou restés cachés ${ }^{34}$;

- des tentatives de correction des textes transmis, ope ingenii (ce qui présuppose la maîtrise d'un assez vaste corpus d'œuvres) ou ope codicum ${ }^{35}$;

- la traduction en latin des œuvres anciennes écrites en d'autres langues, tout d'abord en $\operatorname{grec}^{36}$;

- la transmission et diffusion des sources anciennes grâce à l'imprimerie, des éditions princeps ${ }^{37}$ aux grandes éditions de la fin de la Renaissance, en rappelant combien étroitement les imprimeurs de cette période sont associés aux recherches humanistes et se confondent dans la plupart des cas avec les humanistes eux-mêmes ;

- une étude approfondie du lexique grec et latin, certes pour établir le sens exact de vocables, notamment techniques, déterminants aux fins de l'exégèse, mais aussi afin de rassembler les matériaux pour des commentaires ou des thesauri ${ }^{38}$;

34 Voir en premier lieu R. SABBADINI, Le scoperte dei codici latini e greci ne' secoli XIV e XV [1905-1914], t. I-II, Florence, Sansoni, 1967. Une synthèse toujours utile d'histoire de la philologie classique, comportant un ample développement sur l'Humanisme (où Budé a toute sa place), est celle de Leighton D. Reynolds et Nigel D. Wilson, D'Homère à Érasme. La transmission des classiques grecs et latins [1974], trad. fr., Paris, CNRs Éditions, 1984.

35 Voir les ouvrages classiques de S. Timpanaro, La genesi del metodo del Lachmann [1963], Turin, UTET, 2004, et de J. JeHASSE, La Renaissance de la critique. L'essor de l'humanisme érudit de 1560 à 1614 [1976], Paris, Honoré Champion, 2002.

36 Consulter Mariarosa CORTESI, Silvia FIASCHI, Repertorio delle traduzioni umanistiche a stampa. Secoli XV-XVI, t. I-II, Florence, SISMEL, 2008, outre les neuf volumes déjà parus (1960-2011) du Catalogus translationum et commentariorum, par Paul Oskar KRISTELLER, Ferdinand Edward Cranz, Virginia Brown et alii, Washington, CUA Press.

37 Il est malaisé de dresser une liste complète des princeps grecques et latines, notamment du fait des éditions partielles avant la phase des œeuvres complètes. Voir Beriah Botrield, Prefaces to the first editions of the Greek and Roman classics and of the Sacred Scriptures, Londres, H. G. Bohn, 1861. L'exemple particulièrement significatif d'une étude philologique sur les textes de quelques éditions vénitiennes d'Alde Manuce est offert par Martin SiCHERL Griechischen Erstausgaben des Aldus Manutius. Druckvorlagen, Stellenwert, kultureller Hintergrund, Paderborn, F. Schöning, 1997. Voir en outre, pour les éditions grecques, Émile LEGRAND, Bibliographie hellénique ou description raisonnée des ouvrages publiés par des Grecs aux XVe et XVI siècles [1886-1906], réimpr. Paris, Maisonneuve et Larose, 1962.

38 Pour les commentaires, voir le Catalogus translationum et commentariorum, cité. Parmi les thesauri, citons Niccolò Perotti, Cornu Copia, éd. Jean-Louis Charlet et al., t. I-VIII, Sassoferrato, Studi umanistici piceni, 1987-2001, étonnant fourre-tout encyclopédique prenant pour base les Épigrammes de Martial. Quelque quarante ans avant l'imposant Thesaurus lingua grece d'Henri Estienne (1572), Guillaume Budé publie ses Commentarii lingue Grace (1529 ; infra) et Étienne Dolet ses Commentarii lingue Latine, en deux volumes (Lyon, S. Gryphe, 1536-1538). 
- l'enquête sur divers aspects matériels ou institutionnels de l'Antiquité ${ }^{39}$;

- une approche transdisciplinaire de nature encyclopédique ${ }^{40}$ croisant plusieurs types de sources (historiques, philosophiques, poétiques, oratoires, juridiques, religieuses... $)^{41}$;

- des efforts vers l'étude des langues sémitiques, outre l'hébreu biblique, l'araméen, le syriaque et l'arabe, d'abord - mais non seulement - au service de la connaissance de l'Antiquité et de sa transmission ${ }^{42}$.

Voilà bien des domaines d'étude encore d'actualité au XXI ${ }^{\mathrm{e}}$ siècle.

Bien évidemment, si l'on passe de la considération des contenus à celle des raisons qui motivent les recherches humanistes, une nette différence avec la recherche actuelle apparaîtra : ces savants polyvalents étaient parfois mus, aussi, par des préoccupations bien étrangères à celles des historiens de notre époque et qu'il faut remettre dans leur contexte précis : intérêt pour la kabbale ou pour la connaissance de la géométrie, pour l'habileté oratoire ou pour la pratique de la médecine. Rien de ce qui touche à l'humain

39 Avec Cyriaque d'Ancône (1391-1452), c'est, nous l'avons vu, Flavio Biondo - par ailleurs grand historien avec ses Historia ab inclinatione Romanorum imperii allant $\mathrm{du} \mathrm{v}^{\mathrm{e}}$ au $\mathrm{XV}^{\mathrm{e}}$ siècle - qui fait figure d'initiateur dans ce domaine, grâce à sa trilogie Roma instaurata (sur la topographie de l'Urbs, 1444-46), Italia illustrata (sur les provinces de la Péninsule, 1448-58) et Roma triumphans (sur les institutions politiques et religieuses, 1459). Pour un exemple français autre que Budé, Lazare de Baiff, père du poète Jean-Antoine et premier traducteur en français d'une tragédie (Électre de Sophocle en 1537), publia des études à succès sur les vêtements, les vases et les navires antiques, De re vestiaria (1526), De vasculis (1531) et De re navali (1536). Voir à ce propos L.-A. SANCHI, "Lazare de Baïf (c. 14901547) ", dans In spinis... Turnhout, Brepols, à paraître en 2012.

40 C'est pour désigner un concept cher à son cour que Guillaume Budé a employé en 1508,

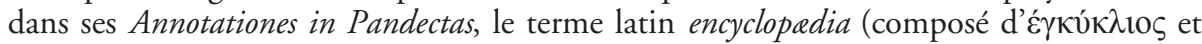
$\pi \alpha 1 \delta \varepsilon i ́ \alpha$, lu sans doute chez Quintilien mais trouvé aussi, par exemple, dans l'édition de 1504 de la Cornu Copia de Perotti, citée), qu'il introduit en français, vers 1519, dans le manuscrit d'un opuscule appelé Institution du prince. Voir G. GUEUDET, " Guillaume Budé, parrain d'encyclopédie ou le vrai texte de l'Institution du prince " in Le Génie de la forme. Mélanges de langue et littérature offerts à Jean Mourot, Nancy, Pun, 1982.

41 Ange Politien a théorisé une telle approche comme typique du savoir souverain du bon philologue (grammaticus) dans sa leçon inaugurale dite Lamia : voir l'édition critique par Ari Wesseling, Angelo Poliziano, Lamia : prelectio in Priora Aristotelis Ananytica, Leyde, E.J. Brill, 1986, et Christopher S. Celenza, Angelo Poliziano's Lamia : text, translation and introductory studies, Leyde, E.J. Brill, 2010. Politien a appliqué extensivement cette méthode dans ses deux Miscellaneorum Centurie (1489-1494).

42 Les cas de Pic de La Mirandole et de Jean Reuchlin sont bien connus, de même que l'appel à l'étude des langues contenu dans la célèbre « Lettre de Gargantua à Pantagruel » (François Rabelais, Gargantua, 1532, ch. VIII). On souhaiterait voir bientôt publiée la thèse de la regrettée Sophie Kessler-MesGuich, Les études hébrä̈ques en France, de François Tissard à Richard Simon (1508-1680), Paris, 1994 ; voir sa contribution sur "L’hébreu chez les hébraïsants chrétiens des XVI ${ }^{\mathrm{e}}$ et XVII ${ }^{\mathrm{e}}$ siècles ", Histoire épistémologie Langage, 18/1 (1996), p. 87-108. 
ou au divin n'était indifférent aux plus curieux d'entre eux. Il appartient dès lors à l'historien de l'humanisme de retracer les itinéraires intellectuels des savants de l'époque et de comprendre les enjeux politiques et culturels qui poussaient ces hommes à étudier et à publier : ce qui arrivera demain aux intellectuels d'aujourd'hui, qui feront à leur tour l'objet d'enquêtes savantes sur la validité des productions scientifiques actuelles.

Esquisser une typologie générale du courant humaniste est relativement aisé. En écrire la longue et luxuriante histoire, offrir un regard panoramique qui en rende compte assez en détail sur les plans chronologique, géographique et de la prosopographie, faisant apparaître les sommités autant que les personnages de seconde zone, relève encore de la gageure tant le phénomène est important en durée, étendue et nombre de personnages à répertorier ${ }^{43}$. L'absence d'un enseignement universitaire spécifique condamne d'ailleurs la Renaissance humaniste à se passer de manuels et d'introductions véritablement générales ${ }^{44}$. Pour construire une vision plus complète, il faudra donc croiser des synthèses diverses, selon les pays et les différents domaines d'étude, sans oublier l'histoire du livre ${ }^{45}$, tout en sachant que bien des points ne sont actuellement traités que dans des études spécifiques, articles ou monographies.

43 Pour se faire une première idée de cette étendue, voir la Bibliographie internationale de l'Humanisme et de la Renaissance, annuelle (à partir de 1965), Genève, Droz, sous le patronage de la FISIER. Entièrement constitué de fiches reproduites, l'instrument de référence en la matière reste, pour l'Italie, Mario Emilio CosEnZA, Biographical and bibliographical Dictionary of the Italian humanists and of the world of classical scholarship in Italy: 1300-1800, t. I-V, Boston, G.K. Hall, 1962. Répertoire plus agile, focalisé sur la notion d'humaniste " transmetteur " d'œuvres anciennes et médiévales, Jean-François MaILLARD, Judit Kecskeméti, Monique Portalier, L'Europe des Humanistes, XIV ${ }^{e}$-XVI ${ }^{e}$ siècles, Brepols - CNRS, Turnhout - Paris, 1995. Les biographies nationales et nommément les volumes du Dizionario biografico degl'Italiani (DBI) restent indispensables pour tout approfondissement bio-bibliographique.

44 Les introductions à la Renaissance privilégient - à juste titre - les arts. Les ouvrages sur l'humanisme traitent surtout de l'aspect littéraire et stylistique : voir Paul VAN TiEghEM, La Littérature latine de la Renaissance. Étude d'histoire littéraire européenne [1944], Genève, Slatkine, réimpr. 1966. Marc FumAROLI a exposé de manière fort stimulante la réduction progressive de la res litteraria à la " littérature " depuis le XVI siècle dans l'introduction à L'Âge de l'Éloquence. Rhétorique et "res literaria " de la Renaissance au seuil de l'époque classique [1980], Genève, Droz, 2002, p. 17-36. Voir aussi Jean-Claude Margolin, Anthologie des humanistes européens de la Renaissance, Paris, Gallimard, 2007, et Jean-Christophe Saladin, Bibliothèque humaniste idéale, de Pétrarque à Montaigne, Paris, Les Belles Lettres, 2008, outre Jacques Chomarat et al., Prosateurs latins en France au XVI siècle, Paris, PUF, 1987, et les deux riches volumes bio-bibliographiques collectifs Centuria Latina réunissant chacun, comme le dit le sous-titre, Cent une figures humanistes de la Renaissance aux Lumières, Colette Nativel (dir.), Genève, Droz, 1997 et 2006.

45 Voir, pour la France, Roger Chartier et Henri-Jean Martin (dir.), Histoire de l'édition française. I : Le livre conquérant [1982], Paris, Fayard - Cercle de la librairie, 1989, en particulier p. 186-302. 
Si le contexte que nous venons de rappeler très brièvement est correctement décrit, les éléments qui le composent forment un ensemble assez cohérent pour en appeler à redonner toute sa place à la production érudite de Budé au sein de la réflexion sur les Antiquaires lancée par Momigliano. Étudier dans ce cadre historique et conceptuel le travail érudit qu'a pu rassembler Guillaume Budé au cours de sa longue vie revêt alors un intérêt d'autant plus grand que cette partie majoritaire de son œuvre n'a toujours pas fait l'objet d'une analyse systématique ${ }^{46}$. Sa production érudite illustre-t-elle l'esprit de l'humanisme européen en ce qu'il a de meilleur à offrir aux amoureux modernes du passé grec et romain ?

Héritier du courant philologique de l'humanisme italien, celui des Valla et Politien, Budé s'ouvre à l'étude de l'Antiquité entre 1491 (retour à l'étude du Digeste) et 1494 (apprentissage du grec ancien) ${ }^{47}$. À ce moment, le patrimoine littéraire grec, après le latin, commence à peine son transfert dans des éditions imprimées, d'abord sous les presses d'Italie mais diffusées à l'échelle européenne, tout en étant encore transmis de façon manuscrite par les derniers copistes et savants post-byzantins. Déjà des pans entiers de littérature grecque étaient connus grâce aux traductions latines du Quattrocento, tandis que les grands auteurs romains étaient accompagnés des premiers commentaires "grammaticaux ». À Paris, Budé a connu et fréquenté le diplomate savant Janus Lascaris et le copiste et professeur Georges Hermonyme de Sparte. Ils ont d'abord été ses maîtres de grec en 1494-1495. Le premier lui a prêté entre 1503 et 1509 une caisse de ses précieux livres, dont le contenu reste inconnu, le second en a copié pour lui plusieurs dizaines ${ }^{48}$. Dans les collèges de la Faculté des arts, il a pu suivre les cours de Jacques Lefevre d'Étaples sur Aristote et sur les sciences exactes, entendre Giovanni Giocondo expliquer le texte de Vitruve ${ }^{49}$, dont celui-ci donnera une remarquable édition en 1511.

46 Les fondateurs de l'association "Guillaume Budé » ne se sont d'ailleurs pas trompés en se réclamant de cet éminent érudit de la Renaissance française, non plus que les générations de lecteurs de la "Collection des Universités françaises " (Paris, Les Belles Lettres), en l'appelant « collection Budé ».

47 Nous proposons ici une synthèse des données biographiques de Budé ; voir la bibliographie donnée en ouverture.

48 Il est très possible que Budé ait demandé à Hermonyme de copier pour lui certains de ces livres de Lascaris. Voir la monographie consacrée à Hermonyme, à son œuvre d'intermédiaire et de copiste et à ses dédicataires, voire possesseurs de ses copies, dont Budé, dans Jean-François Maillard et Jean-Marie Flamand (collab. L.-A. Sanchi, M.-É. Boutroue), La France des Humanistes. Hellénistes, II, Turnhout, Brepols, 2011, p. 1-214.

49 Outre la bibliographie déjà citée, on consultera pour cette période l'ouvrage très fouillé, modèle de son genre, Augustin Renaudet, Préréforme et humanisme à Paris pendant les premières guerres d'Italie (1494-1517) [1916], Paris, Librairie D’Argences, 1953, ainsi 
En ces années politiquement marquées par les premières Guerres d'Italie (14941515), Budé a mené de front son entrée dans la haute fonction publique en devenant secrétaire du roi et ses débuts d'humaniste en traduisant en latin, premier Français moderne, des opuscules grecs de Plutarque et Basile le Grand ${ }^{50}$. Juriste de formation, il entame sa production d'auteur, comme nous l'avons déjà dit, avec la critique philologique et historique du Digeste que renferment ses Annotationes in XXIV Pandectarum libros, parues en 1508 et répondant à l'appel de Valla et Politien à nettoyer le texte du Corpus Iuris ciuilis. Dans les cinq livres De Asse et partibus eius son vaste savoir est mis au service de l'étude des faits économiques de l'Antiquité gréco-romaine et, en petite partie, orientale. Outre les contenus scientifiques, le De Asse témoigne d'une profonde insatisfaction à l'égard des milieux culturels proches de la cour de Louis XII en ses dernières années de règne, cour que Budé a préféré éviter de fréquenter.

La phase qui s'ouvre sous François I $^{\text {er }}$ verra au contraire Budé s'impliquer de plus en plus auprès du roi "Père des lettres ", dans les rôles officiels de conseiller, de bibliothécaire royal et de prévôt des marchands de Paris ${ }^{51}$. Position qui lui permettra de promouvoir l'enseignement universitaire des langues anciennes en France. Est-ce l'effet des exhortations contenues dans la dédicace au roi des Commentarii linguce Grace (1529), qui consacraient Budé comme chef de file des hellénistes d'Europe ? Quelques mois plus tard, des chaires de grec ancien et d'hébreu furent financées, embryon du Collège des lecteurs royaux, futur Collège de France. Si bien que l'institution actuelle fait remonter à l'année 1530 le début de ses activités et affiche cette date jusque dans son logo. Mais en 1535, par suite de l'Affaire des Placards survenue en 1534, Budé sera également obligé de défendre son idée d'humanisme en publiant un opuscule $D e$ Transitu Hellenismi ad Christianismum, face à la répression des ferments luthériens à Paris, ville où imprimeurs, étudiants et professeurs étaient, à tort ou à raison, en butte aux soupçons de la puissante Faculté de théologie, souvent suivie par le Parlement dans ses volontés de censure.

que M. Fumaroli (dir.), Les Origines du Collège de France (1500-1560), Paris, Collège de France - Klincksieck, 1998. La Bibliothèque nationale de France conserve sous la cote "Rés. V. 318 » l'exemplaire de l'édition de Vitruve de Venise, 1497, où Budé a transcrit en forme d'annotations les enseignements de Giovanni Giocondo. Voir à ce propos Lucia A. Ciapponi, "Agli inizi dell'umanesimo francese : Fra Giocondo e Guglielmo Budé », in Forme e vicende. Per Giovanni Pozzi, Padoue, Antenore, 1988, p. 101-118.

50 Voir L.-A. SANCHI, "Budé et Plutarque : des traductions de 1505 aux Commentaires de la langue grecque », in O. Guerrier (dir.), Moralia et Euvres Morales à la Renaissance, Paris, Honoré Champion, 2008, p. 91-108.

51 L'ascension de Budé a probablement été favorisée par le succès international du De Asse. En 1522, Budé en publie un abrégé en français plusieurs fois réédité, le Summaire et Epitome du livre de Asse, sans doute destiné aux milieux de la cour, qui ignoraient le latin. Voir l'édition critique par M.-M. De La Garanderie et L.-A. Sanchi, Paris, Les Belles Lettres, 2008. 
Le De Asse occupe une place particulière dans l'œuvre de Budé comme dans la production antiquaire de son temps. C'est en effet une vaste monographie abordant de multiples aspects de l'histoire ancienne sous un angle unique et bien défini : l'as est à la fois la monnaie concrète et ses subdivisions qui servent en latin à désigner les fractions arithmétiques, par des termes qui renvoient en outre aux systèmes de poids et mesures. L'économie est comprise comme un ensemble de faits - prix, valeurs, frais, dépenses privées ou publiques - étayés dans les sources historiques ou littéraires. Si les tortuosités et les digressions dont s'encombre souvent la prose latine de Budé laissent perplexe le lecteur moderne, la cohérence scientifique du programme réalisé est aussi sûre que la qualité de ses démonstrations et la variété de ses méthodes. Elles vont de la critique textuelle - dont les déductions et impasses sont narrées comme dans un récit d'aventures - à l'expérience directe de comparaison entre poids, mesures, dosages et prix de vente, outre, bien entendu, une petite pratique des monnaies hellénistiques et romaines qu'il a pu recueillir.

Il n'y a pas lieu ici d'entrer dans le détail des contenus ; force est de constater que ce chef-d'œuvre d'historiographie gagnerait à être mieux connu des historiens antiquisants comme des historiens tout court. C'est pour cette raison qu'une édition avec traduction est actuellement en cours ${ }^{52}$; édition critique, elle entend par ailleurs rendre visible le patient travail d'enrichissement et réécriture de son ouvrage auquel s'est livré l'auteur dès l'année 1515, en préparant une sorte de " première édition bis " parue en 1516 et, ensuite, pour des éditions données en 1522, 1524, 1527 et 1532, chacune augmentée ${ }^{53}$ et mise à jour des nouveaux textes grecs disponibles grâce aux nouvelles princeps. Budé poursuit ses recherches sans doute jusqu'à sa mort : la dernière édition augmentée paraît posthume en 1541.

L'accueil d'un tel ouvrage a été enthousiaste à travers l'Europe humaniste, ce dont témoigne la correspondance très internationale et bilingue - plusieurs lettres sont entièrement rédigées en un grec soigné - que Budé s'empresse de publier dès $1520^{54}$. La fortune du De Asse s'étend tout au long du siècle, malgré une polémique autour de la paternité de certaines découvertes sur les systèmes monétaires, qui revenait, d'après les tenants de l'humanisme italien, à Leonardo Porzio, auteur d'un De sestertio publié toutefois en 1520 au plus tôt. L'apport de Budé est par la suite incorporé dans les

52 Voir les deux articles de présentation : L.-A. SANCHI, «Tel un roman philologique. Enjeux d'une édition du De Asse de Budé ", Bibliothèque d'Humanisme et Renaissance, 73 (2011), p. 117-124, et "L'enquête de Budé sur l'économie antique. Notes sur un travail en cours ", Quaderni di storia, 75 (2012), p. 129-147.

53 L'édition de 1522, seule in-quarto au milieu des autres in-folio, parut à Venise des presses d'Alde Manuce, mort en 1515. Jean Grolier, riche émissaire français en poste en Italie et ami des héritiers d'Alde, avait tenté de financer cette édition vénitienne dès 1519 ; voir, sur cet épisode, Anthony Hobson, Renaissance book collecting. Jean Grolier and Diego Hurtado de Mendoza, their books and bindings, Cambridge, C.U.P., 1999, p. 43-45.

54 Voir G. Gueudet, L'art de la lettre humaniste, Paris, Honoré Champion, 2004. 
ouvrages antiquaires, notamment celui de Johann Friedrich Gronovius (1611-1671), De sestertiis sive subsecivorum pecunice veteris Grace et Romana libri IV ${ }^{5}$, où le nom de Budé n'est plus mentionné.

Comme chez les meilleurs savants humanistes, le travail original de Budé s'est fondé sur un dépouillement extensif - sinon systématique - des sources disponibles à Paris à cette époque encore aurorale pour la France, à la différence de l'Italie. Le bilan détaillé de ses lectures personnelles, grecques et latines, au moment où, âgé de plus de soixante ans, il annotait son exemplaire personnel des Commentaires de la langue grecque en vue d'une nouvelle édition, a été fait ${ }^{56}$. Il permet de dénombrer plusieurs dizaines d'auteurs grecs et romains, en grande partie cités si précisément qu'il est difficile de songer à un recours à des sources intermédiaires, d'autant plus qu'il nous reste sept de ses carnets d'extraits variés - pour un total de quelque mille huit cent feuillets - levant partiellement le voile sur sa patiente entreprise, qui a dû s'étendre sur plusieurs décennies ${ }^{57}$.

Le dépouillement des citations du De Asse dans ses rédactions successives étendra nos connaissances sur une étape antérieure aux Commentaires et contemporaine des grandes princeps grecques. Il nous permettra d'identifier, d'une part, les phases de son travail de lecteur omnivore, d'autre part, les manuscrits et imprimés sur lesquels il a pu exercer sa sagacité.

Si la renommée du Budé historien de l'économie a été en quelque sorte dépassée par celle du Budé philologue, auteur des Commentaires, le moment est sans doute venu de redonner sa place à l'historien hors pair que Budé a été, bien loin de la tradition antiquaire fondée sur des compilations. Celle-ci ne s'est en effet bâtie qu'après lui et sur ses recherches, comme sur celles de Flavio Biondo au siècle précédent. Par ailleurs, il est grand temps de montrer en quoi l'helléniste a étayé l'historien (et le juriste !) par l'emploi des sources d'histoire romaine en langue grecque, notamment Plutarque et Strabon, et par l'usage des procédés lexicographiques grecs pour expliquer le sens des termes équivalents latins.

55 Première édition, Deventer, 1643 ; l'édition définitive (Leyde, 1691), posthume, est due à Jakob Gronovius.

56 La deuxième édition des Commentaires, "tertia parte aucti », paraît posthume en 1548 : le travail préparatoire a donc eu lieu entre 1529 et 1540 . L'exemplaire personnel de Budé de la première édition avec ses additions autographes est conservé à la $\mathrm{BnF}$, sous la cote « Rés. X. 67 ». Voir L.-A. SANCHI, Les Commentaires, p. 120-135 et 237-278 ; cette somme du savoir linguistique est constituée à partir d'environ vingt mille citations en grande partie grecques $(90 \%)$, mais régulièrement rapprochées d'exemples en latin. À la bibliographie sur les livres de Budé fournie p. 120, note 1, on pourra désormais ajouter Filippomaria PONTANI, "From Budé to Zenodotus : homeric readings in the European Renaissance ", International journal of the Classical tradition, 14 (2007), p. 375-430.

57 Voir à ce propos L.-A. SANCHI, Les Commentaires, p. 142-146, et la bibliographie citée aux notes 14 et 15 . 
Héritier de la meilleure tradition philologique italienne, Budé a aussi été le chef de file de la puissante tradition helléniste française, celle des Estienne, Turnèbe, Casaubon, Lambin qui se sont reconnus comme ses lointains disciples à travers l'enseignement dispensé par les lecteurs royaux. Il a également fondé la grande tradition historique érudite française, celle des juristes et parlementaires " robins ", souvent "politiques " lors des guerres civiles de la deuxième moitié du XVI ${ }^{\mathrm{e}}$ siècle. À la pointe des études de son temps, il a été justement honoré dans les décennies suivantes qui, de manière tout aussi prévisible, ont pu dépasser ses résultats scientifiques, entraînant l'oubli de ses contributions savantes et sa réduction à une icône nationale. Ce que nous pourrions faire aujourd'hui, c'est lui rendre justice en examinant de près ses productions savantes et en essayant de comprendre en quoi précisément il reste, en dépit des siècles qui nous séparent de lui, un exemple à suivre.

Luigi-Alberto SANCHI

CNRS-IRHT

40 avenue d'Iéna

F-75116 Paris

la.sanchi@irht.cnrs.fr 